\title{
O autocuidado de mulheres transexuais à Luz da Teoria do Déficit de Autocuidado de Orem
}

\author{
Self-care of transsexual women in the light of Orem's Self-Care Deficit Theory \\ Autocuidado de las mujeres transexuales a la luz de la Teoría del Déficit de Autocuidado de Orem
}

Recebido: 22/10/2021 | Revisado: 31/10/2021 | Aceito: 06/11/2021 | Publicado: 10/11/2021

Líscia Divana Carvalho Silva

ORCID: https://orcid.org/0000-0002-3624-6446 Universidade Federal do Maranhão, Brasil E-mail: liscia.divana @ufma.br

Aléxia Marielle Damasceno Padilha

ORCID: https://orcid.org/0000-0003-1360-333X

Universidade Federal do Maranhão, Brasil

E-mail: alexia_mariele@hotmail.com

\begin{abstract}
Resumo
Objetivo: Conhecer o autocuidado de mulheres transexuais à Luz da Teoria do Déficit do Autocuidado de Orem. Método: Estudo com 12 mulheres transexuais do Ambulatório de Sexualidade de um Hospital Universitário do Maranhão, Brasil. Foi utilizada a Escala Appraisal of Self-care Agency (ASA-A) para avaliar a capacidade de autocuidado das participantes. Resultados: Mais da metade das entrevistadas fazem ajustes para manutenção da saúde mediante às mudanças situacionais $(58,3 \%)$, certificam se as ações habituais praticadas para manter-se saudável são boas $(66,7 \%)$, relatam estímulo para realizarem seu autocuidado $(66,7 \%)$ e procuram alternativas mais atuais para se cuidarem (83,3\%). A maioria das mulheres apresenta boa capacidade de autocuidado (66,7\%). Conclusão: Conhecer os significados que mulheres transexuais atribuem ao autocuidado constitui-se relevante pela singularidade na saúde e educação que emergem ao contato com a diversidade e identidade.
\end{abstract}

Palavras-chave: Pessoas transgêneros; Identidade de gênero; Autocuidado; Teoria de enfermagem.

\begin{abstract}
Objective: To know the self-care of transsexual women in the light of Orem's Self-Care Deficit Theory. Method: Study with 12 transsexual women from the Sexuality Outpatient Clinic of a University Hospital of Maranhão, Brazil. The Appraisal of Self-care Agency Scale (ASA-A) was used to assess the participants' self-care ability. Results: More than half of the interviewees make adjustments to maintain health through situational changes $(58,3 \%)$, make sure that the usual actions practiced to stay healthy are good (66.7\%), report stimulus to perform their self-care $(66,7 \%)$ and seek more current alternatives to take care of themselves (83,3\%). Most women have good self-care capacity $(66,7 \%)$. Conclusion: Knowing the meanings that transsexual women attribute to self-care is relevant due to the singularity in health and education that emerge from contact with diversity and identity.
\end{abstract}

Keywords: Transgender people; Gender identity; Self-care; Nursing theory.

\section{Resumen}

Objetivo: Conocer el autocuidado de las mujeres transexuales a la luz de la Teoría del déficit de autocuidado de Orem. Método: Estudio cuantitativo con 12 mujeres transexuales de la Clínica ambulatoria de sexualidad de un Hospital Universitario de Maranhão, Brasil. La escala de evaluación de la agencia de autocuidado (ASA-A) se utilizó para evaluar la capacidad de autocuidado de los participantes. Resultados: Más de la mitad de los entrevistados realizan ajustes para mantener la salud a través de cambios situacionales $(58,3 \%)$, \%), certifican que las acciones habituales practicadas para mantenerse saludable son buenas $(66,7 \%)$, reportan estímulos para realizar su autocuidado $(66,7 \%)$ y buscar alternativas más actuales para cuidarse a sí mismos $(83,3 \%)$. La mayoría de las mujeres tienen una buena capacidad de cuidado personal $(66,7 \%)$. Conclusión: Conocer los significados que las mujeres transexuales atribuyen al autocuidado es relevante debido a la singularidad en salud y educación que surgen del contacto con la diversidad y la identidad.

Palabras clave: Personas transgénero; Identidad de género; Autocuidado; Teoría de enfermería. 


\section{Introdução}

Muitas pessoas vivem uma incoerência entre o sexo biológico e a identidade de gênero, pois não se identificam com o atribuído conforme as suas características genitais. A identidade de gênero equivale ao modo como a pessoa se reconhece dentro dos padrões pré-estabelecidos pela sociedade - homem ou mulher - fazendo a inclusão da terminologia "transexual", que compreende aquela pessoa com uma identidade de gênero oposto ao que lhe foi designado biologicamente (Silva, 2016).

$\mathrm{Na}$ transexualidade, o corpo representa fonte de descontentamento, a pessoa transexual reprova veementemente os seus órgãos genitais externos, aqueles que o identificam como pertencente ao seu sexo biológico, e buscam na terapia de reposição hormonal, cirurgia de resignação do sexo, automutilação, tornar-se um novo alguém, fisicamente falando, uma vez que se identifica em um corpo físico que diverge da sua identidade psicossocial (Sturza, 2015).

Compreender a natureza dos seres humanos, a sua interação com o ambiente e o impacto que essa interação tem na sua vida, ajuda a planejar a prática e a definir quais cuidados melhoram a saúde e o bem-estar das pessoas. A melhoria do cuidado deve ser o principal objetivo de uma teoria de enfermagem (Queiroz, 2014). Quando não capacitado para se autocuidar, o indivíduo apresenta um déficit do autocuidado, sendo indispensável a prestação de cuidados e estimulação a alcançar o autocuidado (Andrade, 2018).

Há necessidade de políticas públicas que tenham como objetivo reduzir o estigma e a discriminação, melhorar o acesso à educação, saúde, inserção no mercado de trabalho, contribuição previdenciária, dentre outros, tornando mais equânime o processo, como ações necessárias para redução das desigualdades e qualidade do cuidado em saúde (Silva, 2020).

Os enfermeiros devem ser capazes de conversar, apoiar e ajudar a orientar aqueles que estão lutando para dar sentido aos sentimentos de disforia de gênero, para que façam escolhas totalmente informadas sobre as decisões, que em alguns casos vão durar ao longo da vida e exigirá tratamento e intervenção contínua (Ion, 2021). O objetivo do estudo é conhecer o autocuidado de mulheres transexuais à Luz da Teoria do Déficit do Autocuidado de Orem.

\section{Metodologia}

Estudo qualitativo, transversal, realizado no Ambulatório de Sexualidade de um Hospital Universitário do Maranhão, Brasil em setembro de 2019, constando de entrevista. Participaram do estudo 12 mulheres transexuais. Utilizou-se a Escala Appraisal of Self-care Agency (ASA-A) para avaliar a capacidade de autocuidado. Foram estabelecidas as frequências absolutas e relativas da caracterização sociodemográfica e econômica das mulheres participantes e das variáveis qualitativas relacionadas a capacidade de autocuidado.

Os caminhos possíveis da pesquisa qualitativa conduzem a diferentes técnicas e instrumentos, assim, há uma demanda de estudo rigorosa e persistente, no sentido de sua elucidação. A sustentação teórica da pesquisa qualitativa está ancorada, fundamentalmente nos alicerces da filosofia, sociologia e antropologia, no contexto de possibilidades, em que emergem teorias diversas (Minayo, Costa, 2018).

A discussão dos resultados foi embasada na Teoria do Déficit do Autocuidado de Orem. O projeto foi encaminhado para o Comitê de Ética em Pesquisa do Hospital Universitário do Maranhão, Brasil, tendo recebido parecer favorável sob o número 2.855.321.

\section{Resultado e Discussão}

Predominaram mulheres transexuais na faixa etária entre 18 e 30 anos - 9 (75,0\%), idade média de 28 anos, cor parda - 7 (58,3\%), ensino médio completo e superior incompleto, ambos - 4 (33,3\%), estudante - 6 (50,0\%), solteira - 10 (83,3\%), agnóstica $-4(33,3 \%)$, renda de 1 a 2 salários mínimos - 6 (50,0\%) que moram com pais e familiares $-6(50,0 \%)$. 
A seguir será apresentada a Figura 1, gráfico que mostra as frequências das variáveis qualitativas da capacidade de autocuidado segundo a Escala Appraisal of Self-care Agency (ASA-A).

\section{À medida que as circunstancias mudam, faço ajustes para manter a minha saúde.}

Na Figura 1, nota-se que uma prevalência de que mais da metade das entrevistadas - 7 (58,3\%) fazem ajustes para manutenção da saúde mediante às mudanças situacionais.

Figura 1 - Gráfico mostrando a distribuição da frequência da variável "À medida que as circunstâncias mudam, faço ajustes para manter a minha saúde". São Luís - MA, 2019.

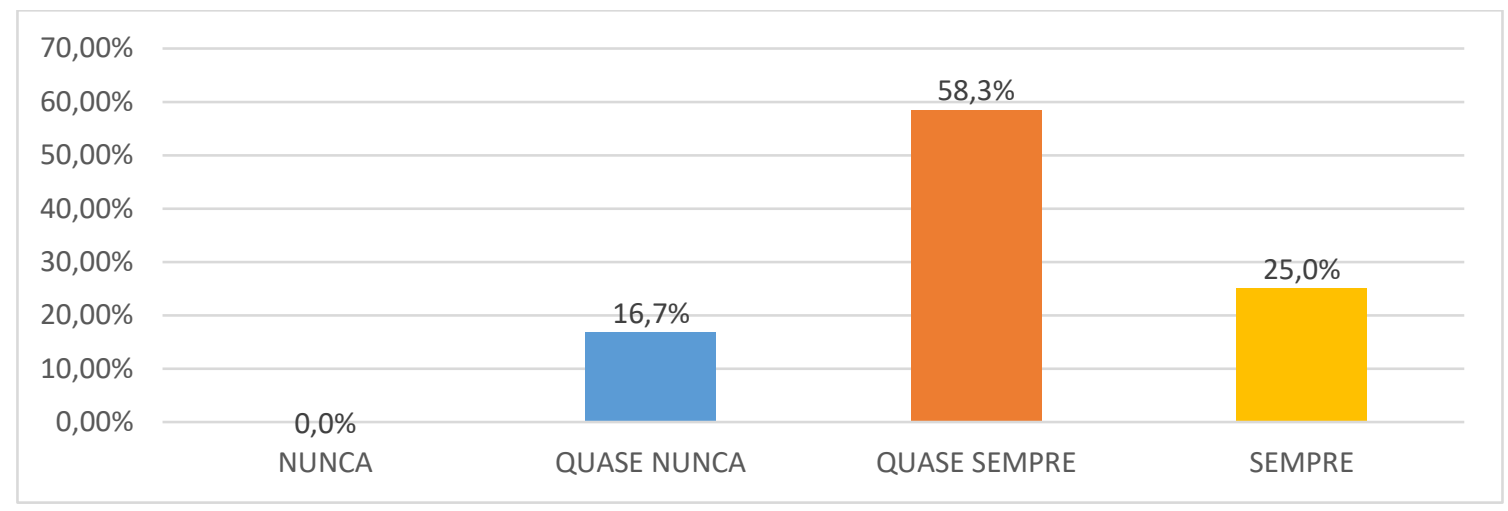

Fonte: Autores (2019).

A capacidade de engajar-se no cuidado consigo mesmo é definido por Orem como uma prática de atividades realizadas deliberadamente que os indivíduos executam para beneficiarem a si, mantendo sua vida, saúde e bem-estar (Foster; Bennetti, 2000). As constantes modificações circunstanciais como à transformação corporal, mudança no processo familiar, situações de violências e discriminação sofridas podem comprometer a capacidade de autocuidado delas.

As mudanças corporais, no início, não são realizadas sob a supervisão de profissionais da saúde, pois são utilizados métodos arriscados como hormônios femininos em doses altas, aplicação de silicone industrial por outras mulheres transexuais mais experientes, entre outros. Além disso, quando as mulheres assumem sua identidade de gênero, em vários casos, culmina na expulsão ou mesmo da evasão voluntária de casa e do convívio familiar. Sem opções de moradia e trabalho formal, é comum inserirem-se no mercado da prostituição (Silva et al., 2016).

Uma vez atuando como profissionais do sexo estão expostas a situações de agressões em geral, assim como infecções sexualmente transmissíveis, nos quais vão interferir na manutenção da saúde dessa população. Diante disso, a enfermagem deve realizar o cuidado no atendimento às suas necessidades de saúde, como orientação e supervisão, capacitação para realizar o autocuidado e no incentivo ao enfrentamento de situações adversas, o que trará repercussões positivas para a manutenção da vida e do bem-estar.

\section{Certifico-me se as formas que pratico habitualmente para me manter com saúde são boas.}

Observa-se na Figura 2, o gráfico mostra que a maior parte das mulheres transexuais - $8(66,7 \%)$ certificam-se se as ações praticadas rotineiramente para manter-se saudável são boas. 
Figura 2 - Gráfico mostrando a distribuição da frequência da variável "Certifico-me se as formas que pratico habitualmente para me manter com saúde são boas". São Luís - MA, 2019.

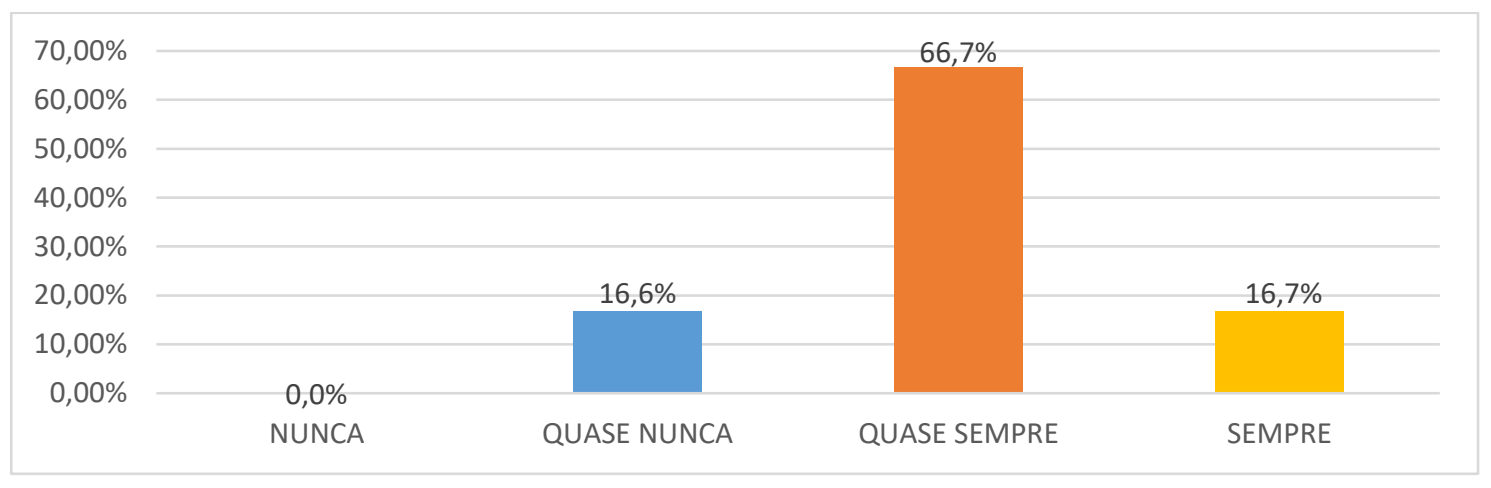

Fonte: Autores (2019).

As pessoas na sua autonomia escolhem o que é melhor pra si e vivem uma constante busca da positividade ou negatividade, aceitando ou rejeitando algo, tornando isso uma autodescoberta incessante (Pacheco; Antunes, 2015). Para mulheres transexuais, a forma de perceber-se saudável é sendo feminina pautada na mudança corporal, no uso de apetrechos femininos, além de procedimentos estéticos (Sampaio, 2013). Os métodos utilizados por essas mulheres para manter-se com saúde e feminina, muitas vezes, não são acompanhados por profissionais qualificados.

É garantido às mulheres transexuais o direito à saúde integral, humanizada e de qualidade no Sistema Único de Saúde (SUS), o que dá direito ao atendimento tanto na rede de atenção básica como em serviços especializados através da Política Nacional de Saúde Integral de Lésbicas, Gays, Bissexuais, Travestis e Transexuais (LGBTT), instituída pela Portaria ${ }^{\circ} 2.836$ de 2011.

O público transexual precisa ser acompanhado, sobretudo, pela atenção primária por meio de atividades de educação em saúde, fortalecendo os laços da prevenção de doenças e agravos à saúde, bem como a garantia de práticas saudáveis voltadas para as mudanças corporais e/ou processo transexualizador. Cabe, aos gestores, subsidiar uma educação permanente para os profissionais inseridos na assistência que, muitas vezes encontram-se despreparados para o atendimento a essa população, assim como a inserção da temática dentro das instituições de ensino que formarão futuros enfermeiros (Silva et al., 2016).

\section{Se eu tiver dificuldade para me mover posso obter ajuda.}

Na Figura 3 apresenta-se o gráfico que mostra - $9(75,0 \%)$ das participantes podem obter ajuda em caso de dificuldades para mover-se. 
Figura 3 - Gráfico mostrando a distribuição da frequência da variável "Se eu tiver dificuldade para me mover posso obter ajuda”. São Luís - MA, 2019.

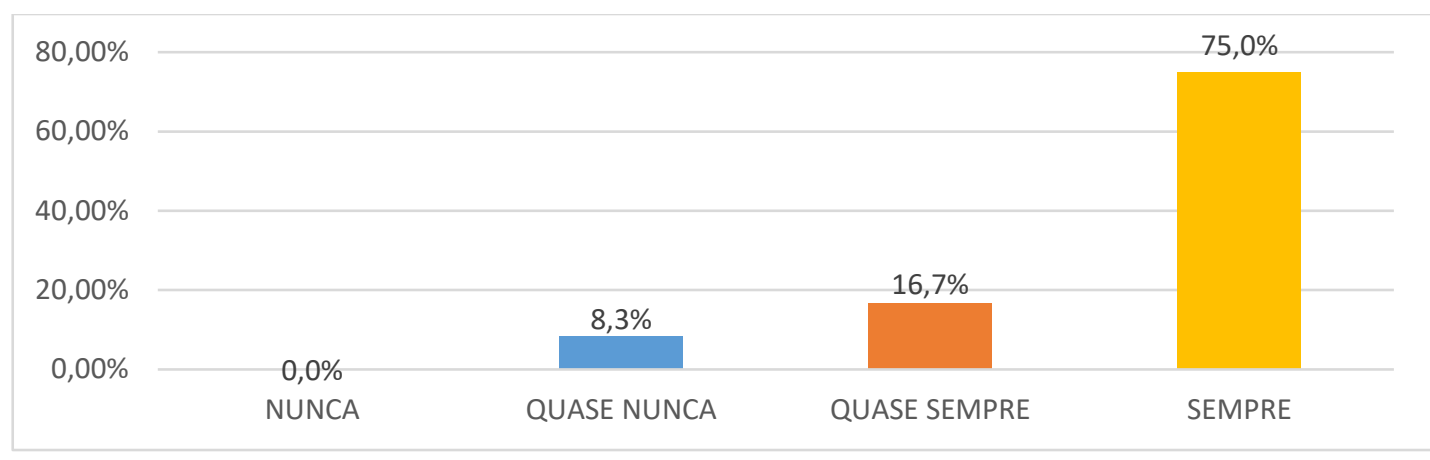

Fonte: Autores (2019).

Só é possível acontecer o autocuidado quando existe um papel ativo do indivíduo, sendo que para a capacidade de se autocuidar as funções humanas básicas são decisórias, e a avaliação dessa habilidade mostrará se esta pessoa está apta para ser independente para realizar seu autocuidado ou se necessita de algum auxílio (Cacciari et al., 2014). Em relação ao apoio social às mulheres transexuais, a literatura mostra, que elas, em sua maioria, são alvo de marginalização e discriminação por vários segmentos da sociedade, sobretudo, pela família e profissionais de saúde (Ferreira, 2018; Monteiro; Brijeiro, 2019).

As mulheres transexuais relatam receber algum tipo de apoio e/ou suporte, quer seja da família nuclear ou mesmo da família extensa, como irmãos, tios, cunhado, e se referem à mãe e aos amigos como principal fonte de auxílio (Soares et al, 2011). A aceitação da identidade de gênero pelos familiares e amigos, torna mais provável que mulheres transexuais recebam cuidados quando incapacitadas fisicamente.

Assim, se existir um déficit entre as ações de autocuidado e as exigências de autocuidado, a enfermagem é solicitada. Diante das dificuldades de acesso da população transexual aos serviços de saúde, faz-se necessário o suporte da enfermagem em situações de déficit do autocuidado. Soluções pertinentes devem ser adotas para a carência de uma educação continuada com foco na promoção da humanização, dignidade e respeito à identidade de gênero das mulheres trans que buscam por ajuda nos serviços de saúde (Rocon et al., 2018).

\section{Eu posso fazer o que é necessário para manter o ambiente limpo onde eu moro.}

Quando questionadas sobre a manutenção do lar - 8 (66,7\%) das participantes afirmam sempre fazer o necessário para manter a limpeza do ambiente onde moram, conforme demonstrado na Figura 4.

Figura 4 - Gráfico mostrando a distribuição da frequência da variável "Eu posso fazer o que é necessário para manter o ambiente limpo onde eu moro”. São Luís - MA, 2019.

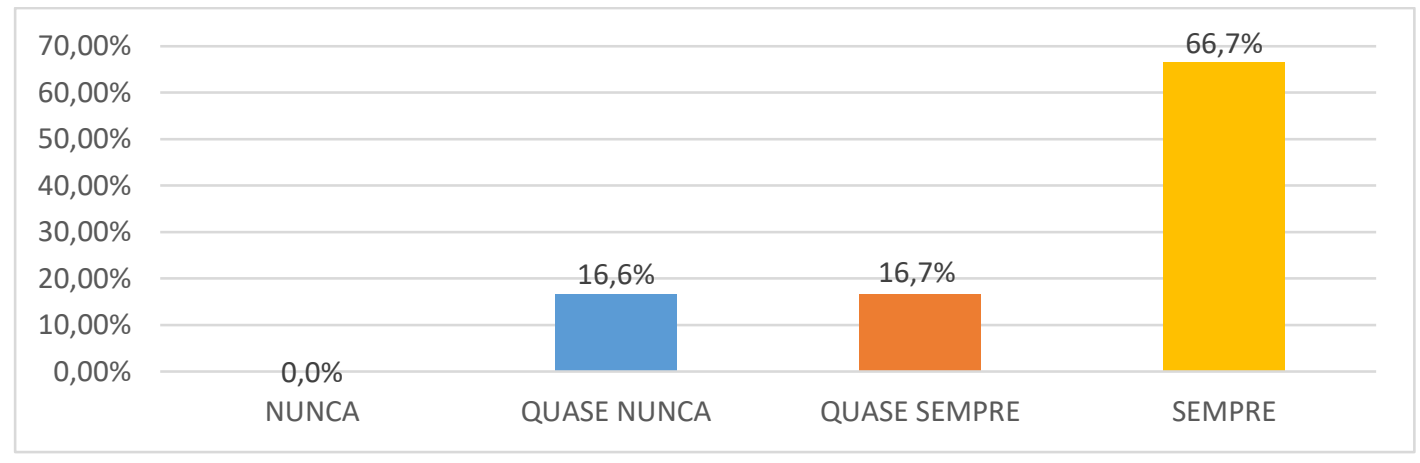

Fonte: Autores (2019). 
Requisitos universais de autocuidado são atividades de um processo de desenvolvimento ou decorrentes de uma nova condição. Tal como a cognição, comunicação, busca do bem-estar pessoal e social, capacidade de gerir riscos físicos, a manutenção do lar e da saúde são demandas imprescindíveis para garantir uma boa qualidade de vida. Ter a capacidade de manter o lar limpo e saudável é uma atividade doméstica que contribui principalmente para manter a pessoa ativa, além de trazer sentimento de utilidade, independência e autoestima, que pode contribuir para um envelhecimento mais ativo e saudável (Nicolato; Couto; Castro, 2016).

O diagnóstico de Enfermagem "Manutenção do lar prejudicada" é definido como "incapacidade de manter, de forma independente, um ambiente seguro para promoção do crescimento". Assim, as participantes do estudo não possuem esta capacidade prejudicada, já que se julgam aptas a realizar a manutenção adequada dos seus lares.

\section{Não tenho força necessária para cuidar de mim como eu deveria.}

A seguir será apresentada a Figura 5 que mostra a frequência da variável força necessária para o cuidar.

Figura 5 - Gráfico mostrando a distribuição da frequência da variável "Não tenho força necessária para cuidar de mim como eu deveria”. São Luís - MA, 2019.

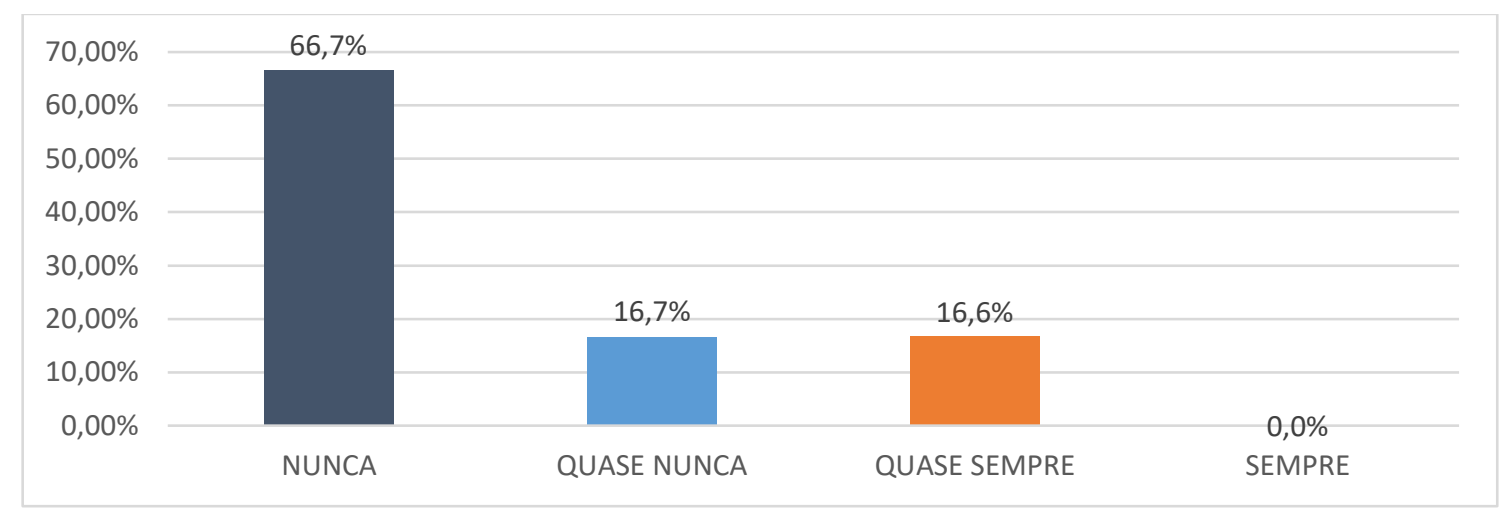

Fonte: Autores (2019).

A capacidade de realizar ações de autocuidado pode sofrer interferência de fatores extrínsecos e intrínsecos, a exemplo: idade, estado de saúde, estado de desenvolvimento, orientação sociocultural, características do atendimento do sistema de saúde, aspectos familiares, padrões de vida, fatores ambientais e adequação situacional ${ }^{5}$. Identificou-se que -8 $(66,7 \%)$ das participantes relatam estímulo para realizarem seu autocuidado, o que pode ser considerado como resultado positivo para o processo de cuidar-se (Figura 5).

Transexuais estão inseridas nas altas taxas de tentativas de suicídio e maiores incidências de desenvolver psicopatologias, além do uso abusivo de substâncias psicoativas (Haas et al., 2011).. O estigma sofrido constantemente configura-se como um fator predisponente para os sofrimentos emocionais em geral e índices elevados de suicídio. A depressão é indicada como o principal transtorno citado entre as transexuais (Asscheman et al., 2011). Desse modo, a terapia hormonal é uma tecnologia que melhora os quadros depressivos, eleva a autoestima, e oferece uma percepção melhor de qualidade de vida (Bauer et al. .2014).

A população transexual/transgênero brasileira continua enfrentando uma batalha bastante árdua. A adoção do nome social segue como um dos principais desafios a serem superados. Essas dificuldades e empecilhos confrontados tornam baixa a 
adesão aos serviços de saúde ofertados e aumentam a demanda de tratamentos fora do sistema, sem acompanhamento profissional (Campos et al., 2020).

A aplicabilidade da teoria de Orem permeia a necessidade da enfermagem quando constatada a incapacidade parcial para mediar o autocuidado. É de competência do enfermeiro detectar déficits de autocuidado e planejar ações que atendam às necessidades e valoração das mulheres transexuais, recebendo um cuidado ético, humanista e integral à saúde física e mental.

\section{Eu posso buscar melhores formas de cuidar da minha saúde do que as que tenho agora.}

A Figura 6 mostra a frequência da variável relacionada a buscar melhores formas de cuidar-se.

Figura 6 - Gráfico mostrando a distribuição da frequência da variável "Eu posso buscar melhores formas de cuidar da minha saúde do que as que tenho agora.”. São Luís - MA, 2019.

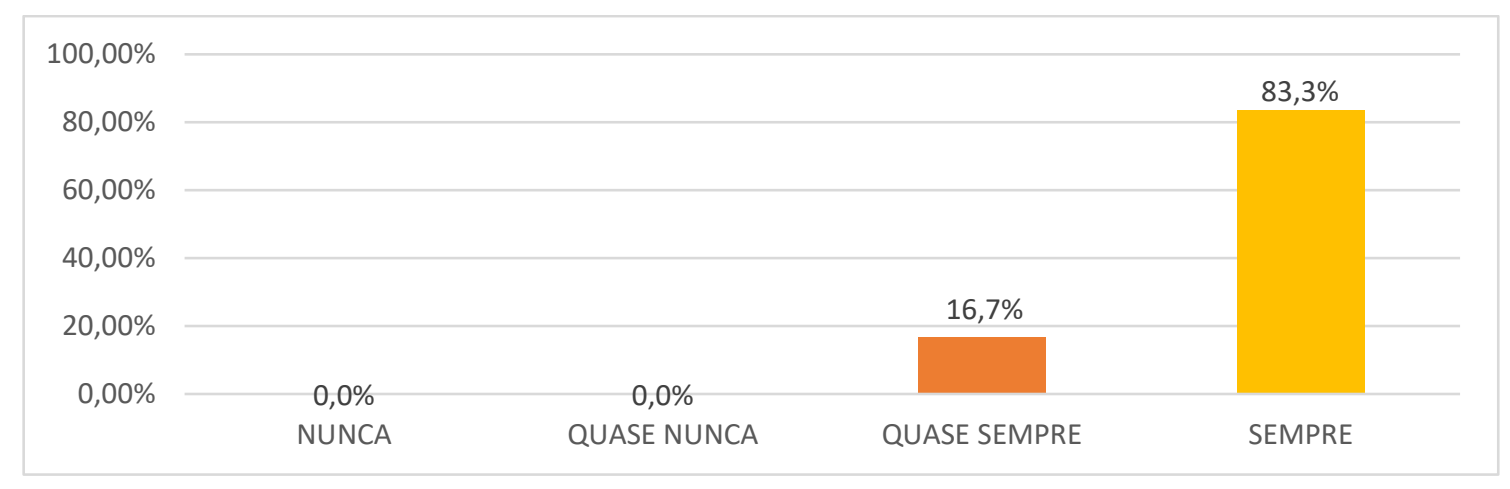

Fonte: Autores (2019).

Observa-se uma prevalência de mulheres transexuais - $10(83,3 \%)$ que procuram por alternativas mais atuais para se cuidar. Ter saúde, para as mulheres transexuais, significa alcançar a transformação do corpo, que é o que tanto desejam, como maneira de eliminar as características que sejam inerentes à identidade de gênero masculina. Assim, elas são motivadas a procurarem informações de maneiras para atingirem o que almejam. A saúde dessa população é produzida no cotidiano, concebendo práticas de saúde de um modo singular (Andrade, 2017).

Portanto, a saúde é construída por várias tentativas de assolar caracteres ambíguos, cuidando e transformando em algo admirável e feminino, apagando os traços masculinos. O uso de hormônios e silicone industrial constituem as principais tecnologias utilizadas para atingir o corpo desejado por essas mulheres, no qual são apontados pelo discurso da área da saúde como práticas de risco e responsáveis pelo desenvolvimento de doenças (Sampaio, 2014).

Torna-se imperativo solicitar das autoridades políticas e aos serviços de saúde um tratamento abrangente, com oferecimento de atendimentos especializados que compreendam as necessidades de transformação do corpo como meio de produção de saúde, assim como os demais aspectos relacionados à estética, social, econômico e cultural, envolvidos na mudança de gênero, que compõe o processo saúde e doença. (Mello et al., 2011).

\section{Quando há situações que me afetam, posiciono-me de forma a não mudar meu jeito de ser}

A seguir será apresentada a Figura 7 mostrando a frequência da variável relacionada a situações que afetam. 
Figura 7 - Gráfico mostrando a distribuição da frequência da variável "Quando há situações que me afetam, posiciono-me de forma a não mudar meu jeito de ser”. São Luís - MA, 2019.

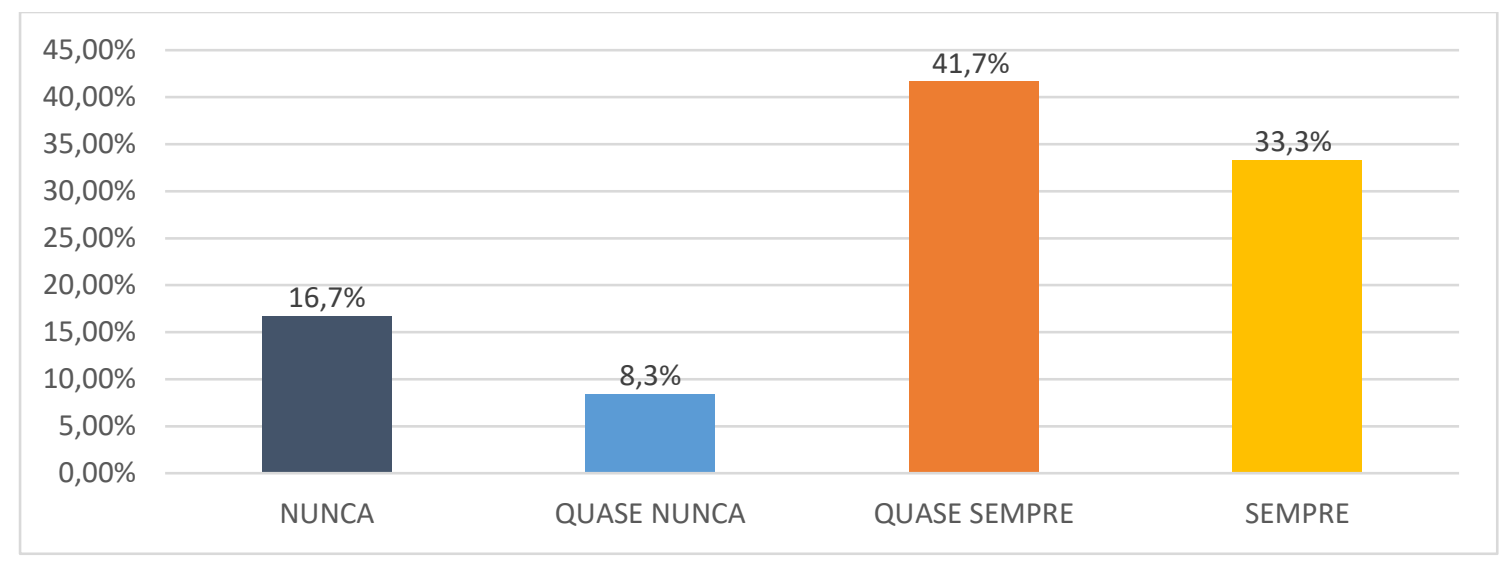

Fonte: Autores (2019).

Quase metade das participantes - $5(41,7 \%)$ expressam que, mediante as circunstâncias que lhes afetam, não mudam a forma de ser, de acordo com o gráfico da Figura 7. À medida que as pessoas conhecem o que é essencial para o seu bem-estar, mais facilmente podem compreender a importância de priorizar o "cuidar de si” nas atividades da vida de modo geral e acima de qualquer situação.

A teoria do déficit de Orem traz que o serviço da enfermagem tem como objetivo transformar o indivíduo totalmente ou parcialmente incapaz em um indivíduo apto para executar o seu autocuidado, além de saber gerenciar fatores ou situações que possam interferir no seu próprio funcionamento e desenvolvimento (Foster \& Bennetti, 2000). Ações de promoção de saúde, muitas vezes não atingem a população como deveria, isso porque os usuários não aderem, por algum motivo, as práticas de bem-estar no seu cotidiano, como a manutenção de hábitos saudáveis. (Mendes, 2010).

A capacidade de autocuidado constitui-se determinante para identificação das potencialidades e limitações das pessoas, a compreender como elas cuidam de si mesmas, além de conhecer as motivações que as levam a submeter-se a procedimentos dolorosos e de riscos (Andrade et al., 2018).

$\mathrm{Na}$ visão das transexuais, a autoestima e sentimentos positivos estão intimamente relacionados à satisfação com a estética corporal e com a saúde psicológica, ou seja, para essas mulheres quanto mais satisfeitas com o próprio corpo, maior a sua autoestima e, por consequência, melhor sua qualidade de vida.

A Tabela 1 abaixo mostra a classificação da capacidade de autocuidado das mulheres transexuais. 
Tabela 1 - Classificação da capacidade de autocuidado das mulheres transexuais do Ambulatório de Sexualidade, São Luís MA, 2019.

\begin{tabular}{lcc}
\hline & N & \multicolumn{1}{l}{} \\
\hline Capacidade de autocuidado & & \\
Baixa capacidade de autocuidado & 0 & 0,0 \\
Regular capacidade de autocuidado & 4 & 33,3 \\
Boa capacidade de autocuidado & 8 & 66,7 \\
\hline Total & 12 & 100,0 \\
\hline
\end{tabular}

Fonte: Autores (2019).

Segundo a escala Appraisal of Self-care Agency (ASA-A) (tabela 1), a maioria das mulheres transexuais - 8 (66,7\%) apresentam boa capacidade de autocuidado.

Para Orem, a atividade de autocuidado é pautada na atuação do indivíduo de forma consciente, intencional e efetiva, alcançando a real autonomia. Já a capacidade de autocuidado não somente é um método que irá garantir a manutenção, reabilitação e benefícios à saúde e bem-estar, mas constitui-se também o desenvolvimento de um potencial para atividade de autocuidado, sendo elemento integrante de cada ser humano. A capacidade de autocuidado se consolida quando o indivíduo pode ou consegue executar o autocuidado e deve aprender a realizar ações de autocuidado terapêutico, em que o enfermeiro mediará emancipação desse indivíduo como protagonista do seu próprio cuidado (Foster; Bennetti, 2000).

\section{Conclusão}

As mulheres transexuais que fazem acompanhamento multidisciplinar no Ambulatório de Sexualidade possuem, na maioria das situações, capacidade para realizarem seu autocuidado. A maioria foi classificada com boa capacidade de autocuidado. Conhecer os significados que mulheres transexuais atribuem ao processo de autocuidado constitui-se relevante para a enfermagem pela singularidade das práticas de saúde e educação que emergem ao contato com a diversidade de sexo e gênero na perspectiva de um cuidado qualificado, pautado nos princípios do Sistema Único de Saúde, especialmente por caracterizar-se pela garantia de atenção à saúde equitativa, integral e universal.

Conhecer os significados que mulheres transexuais atribuem ao processo de autocuidado constitui-se relevante para construção pessoal e profissional; pessoal por permitir compreender a complexidade da vivencia transexual e para a enfermagem pela singularidade das práticas de saúde e educação que emergem ao contato com a diversidade de sexo e gênero na perspectiva de um cuidado qualificado, pautado nos princípios do Sistema Único de Saúde, especialmente por caracterizarse pela garantia de atenção à saúde equitativa, integral e universal.

\section{Referências}

Andrade, C. A., Loureira, A. R., Neto E. R. L., Vasconcelos, E. M. R., \& Araújo, E. C. (2018). Requisitos de autocuidado de mulheres transexuais em uso de hormônios sexuais segundo Teoria de Orem. Cogitare Enferm 23(3): e55748. http://dx.doi.org/10.5380/ce.v23i3.55748.

Asscheman, H., Giltay, E. J. Megens, J. A., Ronde, W. P., Van Trotsenburg, M. A., \& Gooren, L. J. (2011). A long-term follow-up study of mortality in transsexuals receiving treatment with cross-sex hormones. Eur J Endocrinol; 164(4): 635 42. http://dx.doi.org: 10.1530.

Barros, V. A. Associação do risco cardiovascular à capacidade de autocuidado em usuários de drogas [dissertação]. Universidade Federal de Pernambuco. (2015). http://dx.doi.org https://repositorio.ufpe.br/handle/123456789/15903

Bauer, G. R., Scheim, A.I., Deutsch, M. B., \& Massarella, C. (2014). Reported emergency department avoidance, use, and experiences of transgender persons in Ontario, Canada: results from a respondent-driven sampling survey. Ann Emerg Med. 63(6): 713-720. http://doi: 10.1016. 
Research, Society and Development, v. 10, n. 14, e469101422146, 2021

(CC BY 4.0) | ISSN 2525-3409 | DOI: http://dx.doi.org/10.33448/rsd-v10i14.22146

Campos, A. L. O. et al. (2020). Relação Médico Paciente: os desafios na inclusão de pessoas transgêneros e transexuais. Research, Society and Development, 9 (12): 4-13. http://dx.doi.org/10.33448

Cacciari, P., Farias, S. E., Guariente, M. H. D. M., Haddad, M. C. L., \& Martins, E. A. P. (2014); Proposta de autocuidado para trabalhadora readaptada baseado na Teoria de Orem. Rev enferm UFPE on line. 8(5): 1254-1260. 10.5205/reuol.5863-50531-1-ED.0805201420.

Ferreira, D. G. et al. (2018). Meus dias, minha saúde: estudo local sobre a realidade social e os cuidados com saúde de travestis e transexuais em Mato Grosso. Connecton Line, (18), 69-88.

Foster, P., Bennett, A., Dorothea, E., Orem. In: George JB. Teorias de enfermagem: os fundamentos à prática profissional. 4th ed. Porto Alegre: Artmed; 2000. p. 83-102.

Haas, A. P., Eliason, H., Mays, V. M., Mathy, R. M, Cochran, S. D, D'Augelli, A. R, et al. (2011). Suicide and suicide risk in lesbian, gay, bisexual, and transgender populations: review and recommendations. J Homosex ; 58 (1): 10-51.

Homosex, M. 2011 [acesso em 2019 nov 24]; 58(1): 10-51. https://doi: 10.1080 / 00918369.2011.534038.

Ion, R., Patrick, L., Hayter, M., \& Jackson, D. (2021). Sexo, gênero e enfermagem. Journal of Advanced Nursing, 77 (7): 11-12.

Mello, L., et al. (2011). Políticas de saúde para lésbicas, gays, bissexuais, travestis e transexuais no Brasil: em busca de universalidade, integralidade e equidade. Sex Salud Soc, 9, 7-28.

Minayo, M. C. S., \& Costa, A. P. (2018). Fundamentos teóricos das técnicas de investigação qualitativa. Revista Lusófona de Educação, 40 (40): 139-153.

Monteiro, S., \& Brigeiro, M. (2019); Experiências de acesso de mulheres trans/travestis aos serviços de saúde: avanços, limites e tensões. Cad. Saúde Pública. 35 (4): e00111318. https://doi: 10.1590/0102-311X00111318.

NIcolato, F., Couto, A., \& Castro, E. A. (2016). Capacidade de autocuidado de idosos atendidos pela consulta de Enfermagem na Atenção Secundária à Saúde. Enferm. Cent. O. Min., Minais Gerais 6 (2), http://www.seer.ufsj.edu.br/index.php/recom/article/view/1016>.

Pacheco, A., \& Antunes, M. J. (2015). Revisão da literatura sobre motivação para o autocuidado na Atenção Primária em Saúde. Revista Gestão \& Saúde. 6(3): 2907-2918. https://periodicos.unb.br/index.php/rgs/article/view/22422.

Queirós, P. J. P., Vidinha, T. S. S., \& Filho, A. J. A. (2014). Autocuidado: o contributo teórico de Orem para a disciplina e profissão de Enfermagem. Rev. Enf. Ref. 6(3): 157-164 http://dx.doi.org/10.12707/RIV14081.

Rocon, P. C. et al. (2018). O que esperam pessoas trans do Sistema Único de Saúde? Interface, 22 (64): p. 43-63, http://www.scielo.br/pdf/icse/v22n64/18075762-icse-1807-576220160712.pdf>.

Sampaio, J. V., \& Germano, I. M. P. (2017). Tudo é sempre de muito!: produção de saúde entre travestis e transexuais. Estudos Feministas, v 25(2):, 562-65, http://www.scielo.br/pdf/ref/v25n2/1806-9584-ref-25-02-00453.pdf .

Silva, G. W. S., Souza, E. F. L., Sena, R. C. F, Moura, I. B L., Sobreira, M. V. S., Miranda, F. A. N. (2016); Situações de violência contra travestis e transexuais em um município do nordeste brasileiro. Rev Gaúcha Enferm. 37(2): e56407: http://dx.doi. org/10.1590/1983-1447.2016.02.56407.

Silva, M. A., Luppi, C. G., \& Veras, M. A. S. M. V. (2020). Trabalho e saúde na população transexual: fatores associados à inserção no mercado de trabalho no estado de São Paulo, Brasil. Ciênc. Saúde Coletiva 25 (5): 5-10.

Soares, L. (2018). Cuidado em saúde e transfobia: percepções de travestis e transexuais de duas regiões do Rio de Janeiro: Maré e Cidade de Deus, sobre os serviços de saúde [dissertação]. Rio de Janeiro: Escola Nacional de Saúde Pública Sérgio Arouca, fundação Osvaldo Cruz.

Sturza, J. M., \& Schorr, J. S. (2015). Transexualidade e os direitos humanos: tutela jurídica ao direito à identidade. Cesumar. 15(1), 265-283. 